\title{
EQUIVARIANT STABLE STEMS ${ }^{1}$
}

\author{
BY GLEN E. BREDON
}

Communicated by S. Smale, November 14, 1966

Let $S^{n}(r)$ denote the $n$-sphere with a linear involution having a fixed point set of codimension $r$, where $0 \leqq r \leqq n$. We pick some fixed point as a base point and consider the set $\left[S^{n}(r) ; S^{k}(t)\right]$ of base point preserving equivariant homotopy classes of maps from $S^{n}(r)$ to $S^{k}(t)$. This has a natural group structure for $n-r \geqq 1$ and is abelian if $n-r \geqq 2$.

There is a suspension functor $S$ without action and one $\Sigma$ with action (that is, the reduced join with $S^{1}(0)$ and $S^{1}(1)$ respectively). These induce homomorphisms

$$
\left[S^{n+1}(r) ; S^{k+1}(t)\right] \stackrel{S}{\leftarrow}\left[S^{n}(r) ; S^{k}(t)\right] \stackrel{\Sigma}{\rightarrow}\left[S^{n+1}(r+1) ; S^{k+1}(t+1)\right] .
$$

It can be shown that $S$ is an epimorphism when $n \leqq 2 k-1$ and $n-r$ $\leqq 2(k-t)-1$ and is an isomorphism if the strict inequalities hold. Similarly, $\Sigma$ is an epimorphism when $n \leqq 2 k-1$ and $n-r \leqq k-1$ and is an isomorphism if the strict inequalities hold. By passing to the $S$-limit we define

$$
\pi_{n}(r ; t)=\lim _{k}\left[S^{n+k}(r) ; S^{k}(t)\right] .
$$

$\Sigma$ induces $\Sigma: \pi_{n}(r ; t) \rightarrow \pi_{n}(r+1 ; t+1)$ which is an epimorphism when $n \leqq r-1$ and an isomorphism when $n \leqq r-2$. By passing to the $\Sigma$ limit we define

$$
\pi_{n, k}=\lim _{z} \pi_{n}(t+k ; t) .
$$

There is the forgetful functor $\psi$ (forgetting equivariance) and the fixed point set functor $\phi$ which yield homomorphisms

$$
\pi_{n} \stackrel{\psi}{\leftarrow} \pi_{n}(r ; t) \stackrel{\phi}{\rightarrow} \pi_{n-r+t}
$$

where $\pi_{n}$ denotes the classical $n$-stem. For the doubly stable groups these become

$$
\pi_{n} \stackrel{\psi}{\leftarrow} \pi_{n, k} \stackrel{\phi}{\rightarrow} \pi_{n-k} .
$$

1 This research was supported in part by National Science Foundation grant GG-3990 and by a Sloan fellowship. 
It is important to consider the generalization of these groups defined by

$$
\pi_{n}(r, q ; t)=\lim _{k}\left[S^{n+k}(r) / S^{n+k-r+q}(q) ; S^{k}(t)\right]
$$

and we single out the case $q=0$ by the special notation

$$
\pi_{n}^{*}(r ; t)=\pi_{n}(r, 0 ; t) \text {. }
$$

If $r \geqq q \geqq p$ there is an exact sequence

$$
\cdots \rightarrow \pi_{n}(r, q ; t) \stackrel{\gamma}{\rightarrow} \pi_{n}(r, p ; t) \stackrel{\rho}{\rightarrow} \pi_{n-r+q}(q, p ; t) \stackrel{\delta}{\rightarrow} \pi_{n-1}(r, q ; t) \rightarrow \cdots
$$

and also a similar exact sequence with $p$ deleted. A special case of interest is that for which $q=r-1$ :

$$
\cdots \rightarrow \stackrel{\alpha}{\rightarrow} \pi_{n}(r, p ; t) \stackrel{\beta}{\rightarrow} \pi_{n-1}(r-1, p ; t) \stackrel{\psi}{\rightarrow} \pi_{n-1} \stackrel{\alpha}{\rightarrow} \pi_{n-1}(r, p ; t) \rightarrow \cdots
$$

( $p$ may be deleted here). Also of interest is the case in which $p$ is deleted and $q=0$ :

$$
\cdots \rightarrow \stackrel{*}{*}(r ; t) \stackrel{i}{\rightarrow} \pi_{n}(r ; t) \stackrel{\phi}{\rightarrow} \pi_{n-r+t} \stackrel{\Delta}{\rightarrow} \pi_{n-1}^{*}(r ; t) \rightarrow \cdots
$$

(The maps $\psi$ in (5) and $\phi$ in (6) are the forgetful and fixed point homomorphisms respectively.)

The $\Sigma$-suspension yields an isomorphism

$$
\Sigma: \pi_{n}(r, q ; t) \stackrel{\approx}{\rightarrow} \pi_{n}(r+1, q+1 ; t+1)
$$

in all cases.

Let $\Phi(n)$ be the number of integers $k$ with $0<k \leqq n$ and $k \equiv 0,1,2$ or 4 (modulo 8 ). Our main general result is:

TheOREM. If $j$ is divisible by $2^{\Phi(r-q-1)}$ then there is an isomorphism

$$
\lambda_{j}: \pi_{n}(r, q ; t) \cong \pi_{n}(r, q ; t+j) .
$$

If $2^{\Phi(r-q)} \mid j$ then $\lambda_{j}$ commutes with $\psi$. In particular,

$$
\pi_{n}^{*}(r ; t) \approx \pi_{n}^{*}(r ; t+j) \quad \text { for } 2^{\Phi(r-1)} \mid j
$$

and

$$
\pi_{n, k}^{*} \approx \pi_{n, k-j}^{*} \quad \text { for } 2^{\Phi(n+1)} \mid j
$$

Moreover, the period $2^{\Phi(n+1)}$ for $\pi_{n, k}^{*}$ is best possible when $n+1 \equiv 0,1$, 2, 4 (modulo 8). 
Using this result, (7), Spanier-Whitehead duality and results of Atiyah [1] and James [2] it is easy to show that there is an isomorphism

$$
\pi_{k, r}^{n} \approx \pi_{n}^{*}(r ; r+k) \quad \text { when } n<k-1 .
$$

Here $\pi_{k, r}^{n}=\pi_{k+n}\left(V_{k+r, r}\right)$ where $V_{k+r, r}$ is the Stiefel manifold $O(k+r) / O(k)$. In particular, if $n<k-1$ and $n<r-1, \pi_{k, r}^{n} \approx \pi_{n,-\boldsymbol{k}}^{*}$. The periodicity, in $k$, of the $\pi_{k, r}^{n}$ which results from (8) is known and is due to James [2]. Also see [3].

The groups $\pi_{k, r}^{n}$ have been calculated by Paechter [4] for $n \leqq 5$. Our methods, which are aided by the relationships between $\pi_{n}^{*}(r ; r+k)$ and $\pi_{n}(r ; r+k)$, allow us to calculate the groups $\pi_{n}^{*}(r ; r+k)$ for $n \leqq 8$ (with a few ambiguities) and their orders for $n \leqq 10$.

Also the homomorphisms $\psi$ and $\phi$ are determined in roughly this range. We shall comment here only on $\phi$. First, there is the general result:

Proposition. $\phi: \pi_{n, k} \rightarrow \pi_{n-k}$ is onto if $n \geqq 2 k$. It is also onto, and splits, if $k \leqq 0$. If $n<0, \phi$ is an isomorphism.

The calculations yield the following special results:

$$
\begin{aligned}
\phi: \pi_{n, n} & \stackrel{\widetilde{\rightarrow}}{\rightarrow} 2^{n} \pi_{0} \quad \text { for } 1 \leqq n \leqq 4, \\
& \approx: \pi_{5,5} \\
\operatorname{Im}\left\{\phi: \pi_{n, n}\right. & \left.\rightarrow \pi_{0}\right\} \subset 16 \pi_{0} \quad \text { for } n \geqq 4 .
\end{aligned}
$$

There are also exact sequences:

$$
\begin{aligned}
& 0 \rightarrow Z_{2} \rightarrow \pi_{6,6} \stackrel{\phi}{\rightarrow} 16 \pi_{0} \rightarrow 0, \\
& 0 \rightarrow Z_{2} \oplus Z_{2} \rightarrow \pi_{7,7} \stackrel{\phi}{\rightarrow} 16 \pi_{0} \rightarrow 0 . \\
& 0 \rightarrow Z_{16} \rightarrow \pi_{8,8} \stackrel{\phi}{\rightarrow} 16 \pi_{0} \rightarrow Z_{2} .
\end{aligned}
$$

For $n-k=1,2,3$ we obtain

$$
\begin{aligned}
& \phi: \pi_{n, n-1} \rightarrow \pi_{1} \text { is }\left\{\begin{array}{l}
\text { onto for } n \leqq 3, \\
\text { zero for } n \geqq 4 ;
\end{array}\right. \\
& \phi: \pi_{n, n-2} \rightarrow \pi_{2} \text { is }\left\{\begin{array}{l}
\text { onto for } n \leqq 6, \\
\text { zero for } n \geqq 7,
\end{array}\right.
\end{aligned}
$$




$$
\phi: \pi_{n, n-3} \rightarrow \pi_{3} \text { is }\left\{\begin{array}{l}
\text { onto } \pi_{3} \text { for } n \leqq 7, \\
\text { onto } 2 \pi_{3} \text { for } n=8, \\
\text { onto } 4 \pi_{3} \text { for } n=9, \\
\text { onto } 8 \pi_{3} \text { for } n \geqq 10 .
\end{array}\right.
$$

The kernel of $\phi$ can be calculated in the range $n \leqq 8$. (As far as we are aware the only previously known result in this direction was that $\phi: \pi_{n, n} \rightarrow \pi_{0}$ has image contained in $2 \pi_{0}$ for $n \geqq 1$; known since it is an easy consequence of Smith theory.)

The calculations of the $\pi_{n, k}$ are accomplished mainly through the use of spectral sequences associated with the exact sequence (4).

The only difficulties in calculating the $\pi_{n, k}$ are in dealing with the 2-primary components. In fact, if $\mathfrak{e}$ denotes the class of finite 2groups we can prove that

$$
\begin{gathered}
\phi: \pi_{n, k} \stackrel{\approx}{\rightarrow} \pi_{n-k} \bmod \mathcal{C} \text { if } k \text { is odd, } \\
\phi \oplus \psi: \pi_{n, k} \stackrel{\approx}{\rightarrow} \pi_{n-k} \oplus \pi_{n} \bmod \mathcal{C} \text { if } k \text { is even. }
\end{gathered}
$$

An interesting corollary of the proof of the periodicity theorem is worth mentioning here. Let $T_{k}$ be the matrix

$$
\left(\begin{array}{cc}
-I_{k} & 0 \\
0 & I
\end{array}\right) \in O=\bigcup_{m=1}^{\infty} O(m) .
$$

Consider the antipodal involution $A$ on $S^{n}$ and the left translation by $T_{k}$ on $O$. Then we can show that an equivariant map

$$
\left(S^{n}, A\right) \rightarrow\left(O, T_{k}\right)
$$

exists if and only if $2^{\Phi(n)} \mid k$. The "if" part is easy and, in fact, when $2^{\Phi(n)} \mid k$ we construct an equivariant map

$$
\left(S^{n}, A\right) \rightarrow(O(k),-I) .
$$

The proofs of these results will be published elsewhere.

Note added in proof. It has been brought to our attention that the groups $\pi_{k, r}^{n}$ have been calculated for $n \leqq 13$ and $r$ large by C. S. Hoo and M. E. Mahowald. Their results are tabulated in Bull. Amer. Math Soc. 71 (1965), 661-667. Unfortunately, it does not appear that their methods could give any information on the fixed point homomorphism. However, a comparison of their results with our methods does strongly indicate the conjecture that the image of $\phi: \pi_{n, n} \rightarrow \pi_{0}$ is $a_{n} \pi_{0}$ where $a_{8}=a_{9}=2^{5}, a_{10}=2^{6}, a_{11}=2^{7}, a_{12}=a_{13}=a_{14}=2^{8}$. (For $n \leqq 7, a_{n}$ is given above.) 


\section{REFERENCES}

1. M. F, Atiyah, Thom complexes, Proc. London Math. Soc. 11 (1961), 291-310.

2. I. M. James, Spaces associated with Stiefel manifolds, Proc. London Math. Soc. 9 (1959), 115-140.

3. M. Mahowald, $A$ short proof of the James periodicity of $\pi_{k+p}\left(V_{k+m, m}\right)$, Proc. Amer. Math. Soc. 16 (1965), 512.

4. G. F. Paechter, The groups $\pi_{r}\left(V_{n, m}\right)$. (I)-(V), Quart. J. Math. Oxford Ser. (2) 7 (1956), 249-268; 9 (1958), 8-27; 10 (1959), 17-37 and 241-260; 11 (1960), 1-16.

University of California, Berkeley and

The Institute for Advanced Study 